Article

\title{
Performance and Milk Composition of Nubian Goats as Affected by Increasing Level of Nannochloropsis oculata Microalgae
}

\author{
Ahmed E. Kholif ${ }^{1, *}$, Gouda A. Gouda ${ }^{1}$ and Hatem A. Hamdon ${ }^{2}$ \\ 1 Dairy Science Department, National Research Centre, 33 Bohouth St. Dokki, Giza 12622, Egypt; \\ gagouda@gmail.com \\ 2 Department of Animal Production, Faculty of Agriculture, New Valley University, New Valley 72511, Egypt; \\ hamdon@nv.aun.edu.eg \\ * Correspondence: ae_kholif@live.com or ae.kholif@nrc.sci.eg
}

Received: 28 November 2020; Accepted: 18 December 2020; Published: 21 December 2020

Simple Summary: Recently, microalgae, natural marine resources, have gained increasing interests as a feed for animals. Microalgae are single-cell microorganisms that have been used to provide nutrition to humans and animals for centuries. Research has shown that inclusion of microalgae in diets improved feed utilization, milk production and quality, growth performance, and meat quality in ruminants, as a result of improved diet nutritive value leading to improved feed utilization. Very low doses of microalgae in feed enhance growth and lactational performance of ruminants. Nannochloropsis oculata microalgae is a rich source of rumen protected healthy fatty acids which can be explored as a feeding strategy to enhance the nutritional value of milk for consumers.

\begin{abstract}
Fat supplementation affects the lactational performance of goats and dramatically changes milk nutritive value. In the present experiment, two levels of Nannochloropsis oculata microalgae, a natural source of rumen-protected eicosapentaenoic acid (EPA), were studied in the diet of Nubian goats. Using quintuplicated $3 \times 3$ Latin square design, fifteen lactating goats, $(14 \pm 2$ months old and $33.0 \pm 1.3 \mathrm{~kg}$ ) after kidding, were randomly assigned into three treatments in an $84-\mathrm{d}$ assay. Goats were offered a basal diet comprising berseem clover, wheat straw and concentrates in 3:2:5, respectively, (control treatment-no supplementation). The other two treatments were supplemented with $N$. oculata microalgae at $5 \mathrm{~g}$ (NOM5 treatment) or $10 \mathrm{~g}$ (NOM10 treatment)/doe/d. Without affecting intake, treatments improved $(p<0.01)$ nutrient digestibility. Supplementations had no effect on ruminal $\mathrm{pH}$ and ammonia-nitrogen, however, NOM5 and NOM10 linearly improved $(p<0.05)$ total volatile fatty acids and propionic acids. N. oculata supplementation linearly increased $(p<0.01)$ milk yield and lactose content. Supplementation reduced atherogenic index $(p=0.004)$ and enhanced the concentrations of unsaturated fatty acids and C20:5n3 (EPA). Conclusively, feeding Nubian goats on diet supplemented with $N$. oculat $a$ at 5 and $10 \mathrm{~g}$ improved milk production and the nutritive value. No improvements in the performance were observed when N. oculata dose was increased from $5 \mathrm{~g}$ to $10 \mathrm{~g} / \mathrm{doe}$; thus, $5 \mathrm{~g}$ dose is recommended for use.
\end{abstract}

Keywords: blood metabolites; digestibility coefficients; eicosapentaenoic acid; milk fatty acids; lactational performance; Nannochloropsis oculata microalgae

\section{Introduction}

Microalgae are characterized with an extreme rapid growth rate [1], where a large production can be produced in marginal or non-arable land, making their use as feeds to improve food security 
applicable [2,3]. The main disadvantage is the high production cost [4], making them an uncompetitive feed option [3,5]. Due to the technical development, the situation may change in the near future.

Some microalgae are rich in unsaturated fatty acids (UFA). This makes them as options to improve ruminal fermentation and feed digestion [6-8], with high concentrations of docosahexaenoic acid (DHA) and conjugated linoleic acid (CLA) fatty acids in milk [6,9]. Moreover, some other microalgae are richly endowed with n-3 poly UFA, such as $\alpha$-linolenic acid and eicosapentaenoic acid (EPA) $[3,10,11]$. Including microalgae in the diet of ruminants showed promising results $[3,6,7,12]$.

Nannochloropsis is a rich source of rumen-protected EPA, DHA and CLA as well as all essential amino acids required for animal feed $[6,10,13]$. Alves et al. [11] reported that Nannochloropsis is a natural dietary source of EPA for ruminants. Durmic et al. [14] reported that Nannochloropsis contains EPA and DHA (21.5 and 3.2\% fat, respectively). Archibeque et al. [13] evaluated the nutritive value of Nannochloropsis oculata compared with soybean meal and steam-flaked corn, and observed excellent nutritive value as acceptable protein and mineral supplements; however, feeding trials are recommended to validate animal acceptance, digestibility and performance.

Gomaa et al. [6] reported that $N$. oculata microalgae enhanced the ruminal fermentation of feeds, degradability and decreased methane $\left(\mathrm{CH}_{4}\right)$ releasing in vitro. Additionally, Wild et al. [10] reported that EPA (C20:5n-3), the main fatty acids in Nannochloropsis, appears to be a valuable alternative feed product. High EPA concentration has health benefits, reducing both cardiovascular disease and cancer risk [15]. Additionally, the ratio of omega-3/omega- 6 is about 2.7 and can be used as a source of omega-3 fatty acids [10]. Glover et al. [16] and Vahmani et al. [17] noted dietary supplementation of lactating cows with EPA and DHA (i.e., poly UFA) rich microalgae increased milk content of poly UFA.

The Nubian goat is a dual-purpose goat that carries more flesh than other dairy breeds. It produces milk with about 3.5-8\% fat [18]. Therefore, we used it as a model for lactating goats to evaluate N. oculata microalgae supplementation. Information on the effect of supplementing N. oculata microalgae to lactating animals is limited. Therefore, the current study evaluated the effect of supplementing N. oculata microalgae to lactating Nubian goats on feed utilization, ruminal fermentation and milk production, composition, and nutritive value. Our hypothesis was that $N$. oculata is a source of naturally ruminal protected long chain n-3 fatty acids. Moreover, $N$. oculata supplementation would alter feed digestion and fermentability resulting in improved milk production and increased concentrations of milk UFA, omega-3 and CLA.

\section{Materials and Methods}

\subsection{Goats and Experimental Design}

Does care and handling were as outlined in the Guide for the Care and Use of Agricultural Animals in Agricultural Research and Teaching (Federation of Animal Science Societies, Champaign, IL, USA) and approved by the Institutional Animal Care and Use Committee of the Faculty of Agriculture, New Valley University, New Valley, Egypt.

Fifteen multiparous lactating Nubian goats, $14 \pm 2$ months old and weighing $33.0 \pm 1.3 \mathrm{~kg}$ (7 days in milk), were used in a quintuplicated $3 \times 3$ Latin square design experiment ( 3 treatments, 3 periods, 5 does per treatment (resulting in 15 replicates/treatment)). Animals were housed individually in soil-surfaced pens $\left(1.5 \mathrm{~m}^{2} /\right.$ doe) with water and diets supplied ad libitum. Does were fed according to NRC [19] recommendations on a diet containing concentrates feed, berseem clover (Trifolium alexandrinum) and wheat straw (Triticum aestivum) at 5:3:2, respectively, (Table 1).

Goats in all treatments were offered the same basal diet without supplementation (Control treatment), supplemented with $5 \mathrm{~g}$ (NOM5 treatment) or $10 \mathrm{~g}$ (NOM10 treatment) of $N$. oculata/doe/d. To ensure that the whole dose was received, the microalgae was added to the concentrate fraction of the diets during the morning feeding. Does were fed individually two times daily (08:00 and 16:00 h) in two equal proportions. Each experimental period lasted 28 days (comprised two weeks of adjustment to the diet and two weeks of measurements and sample collection). 
Feeds were sampled daily, composited weekly, dried at $60{ }^{\circ} \mathrm{C}$ in a forced-air oven for $48 \mathrm{~h}$ [20]. After, samples were ground to pass a 1-mm screen in a mill and stored for further chemical analyses.

Table 1. Chemical composition of experimental control diet fed to the Nubian goats $(\mathrm{g} / \mathrm{kg}$ DM basis unless otherwise stated).

\begin{tabular}{ccccc}
\hline & $\begin{array}{c}\text { Concentrate } \\
\text { Feed Mixture }\end{array}$ & $\begin{array}{c}\text { Berseem } \\
\text { Clover }\end{array}$ & $\begin{array}{c}\text { Wheat } \\
\text { Straw }\end{array}$ & Control (Basal) Diet ${ }^{2}$ \\
\hline Dry matter (g/kg wet material) & 907 & 214 & 904 & 699 \\
Organic matter & 895 & 858 & 912 & 887 \\
Crude protein & 154 & 144 & 37 & 128 \\
Ether extract & 44.0 & 49.9 & 10.7 & 39.1 \\
Non-structural carbohydrate & 448 & 213 & 116 & 311 \\
Neutral detergent fiber & 249 & 451 & 748 & 409 \\
Acid detergent fiber & 140 & 348 & 505 & 275 \\
Cellulose & 115 & 296 & 368 & 220 \\
Hemicellulose & 109 & 104 & 243 & 134 \\
\hline
\end{tabular}

${ }^{1}$ Consisted of $55 \%$ corn, $25 \%$ wheat bran, $17 \%$ soybean meal, $2 \%$ limestone, $0.5 \% \mathrm{NaCl}$ and $0.5 \%$ mixture of minerals and vitamins. ${ }^{2}$ Control basal diet comprising (/kg DM): $500 \mathrm{~g}$ of concentrates feed, $300 \mathrm{~g}$ berseem clover and $200 \mathrm{~g}$ wheat straw.

\subsection{Nannochloropsis Oculata Microalgae}

As previously explained in Gomaa et al. [6], lyophilized N. oculata biomass produced using BG-II growth medium [21] was used. N. oculata contained $92.2 \%$ dry matter (DM), $81.1 \%$ organic matter (OM), $29.2 \%$ crude protein (CP), $11.1 \%$ carbohydrates, and $29.2 \%$ oil. Chemical and fatty acids analysis of N. oculata have been described in details in Gomaa et al. [6]. Fatty acids profile of N. oculata microalgae is shown in Table 2.

Table 2. Fatty acids profile of N. oculata microalgae.

\begin{tabular}{cc}
\hline Item & $\mathbf{g} / \mathbf{k g}$ Total Fatty Acids \\
\hline C12:0 & 3.1 \\
C14:0 & 72.2 \\
C15:0 & 12 \\
C16:0 & 205 \\
C16:1 & 202 \\
C16:2 & 104 \\
C17:0 & 11 \\
C18:0 $18: 1$ & 22 \\
C18:2 (omega-6) & 33 \\
C18:3 (omega-3) & 34 \\
C20:5n-3 & 11 \\
C22:0 & 283 \\
Total saturated fatty acids (SFA) & 4.1 \\
Total monounsaturated fatty acids & 329 \\
Total polyunsaturated fatty acids & 236 \\
Total unsaturated fatty acids (UFA) & 432 \\
UFA/SFA & 667 \\
\hline
\end{tabular}

\subsection{Digestibility and Chemical Analysis}

During the last two weeks of each experimental period, a digestibility trial was conducted (i.e., 3 digestibility trials). During the measurement weeks, daily feed intake was measured (the difference between served diets and orts from the previous day). Total fecal output was collected, twice daily for each goat before feeding (2 times), and stored at $-10{ }^{\circ} \mathrm{C}$ for subsequent analysis. Daily fecal sample (100 g/does) was taken and pooled within a period. Feeds, orts and feces were 
grounded and analyzed for DM, ash, $\mathrm{N}$ and ether extract according to AOAC [20] official methods. Neutral detergent fiber (NDF) and acid detergent fiber (ADF) were determined using ANKOM $^{200}$ Fiber analyzer and expressed without residual ash. Non-structural carbohydrates (NSC), OM, cellulose and hemicellulose were calculated.

\subsection{Ruminal Fermentation}

Does were sampled individually for ruminal contents $3 \mathrm{~h}$ after being fed in the morning on the last day of each experimental period using a stomach tube and hand pump. The $\mathrm{pH}$ of the ruminal fluid was taken immediately with a $\mathrm{pH}$ meter (HI98127 $\mathrm{pHep}{ }^{\circledR} 4 \mathrm{pH} /$ Temperature Tester, Hanna ${ }^{\circledR}$ Instrument, Villafranca padovana PD, Italy). Rumen contents were strained through four layers of cheesecloth. Approximately $5 \mathrm{~mL}$ of the strained rumen liquor was preserved in $5 \mathrm{~mL}$ of $0.2 \mathrm{M}$ $\mathrm{HCl}$ for the ammonia- $\mathrm{N}\left(\mathrm{NH}_{3}-\mathrm{N}\right)$ analysis using AOAC [20] method. Another $0.8 \mathrm{~mL}$ of the strained rumen liquor was mixed with $0.2 \mathrm{~mL}$ of a solution containing $250 \mathrm{~g}$ of metaphosphoric acid/L for the total volatile fatty acids (VFA) determination by titration. The concentrations and molar proportions of individual VFAs were determined by gas-liquid chromatography (model 5890, Hewlett-Packard, Little Falls, DE, USA).

\subsection{Blood Measurements}

Does were sampled for blood $4 \mathrm{~h}$ after morning feeding. Approximately $10 \mathrm{~mL}$ of blood was obtained from the jugular vein of each doe in a clean dry tube without anticoagulants. After, samples were centrifuged at $4000 \times g$ for 20 min before storing in 2-mL Eppendorf tubes at $-20^{\circ} \mathrm{C}$ pending analysis. By using specific kits (Stanbio Laboratory, Boerne, TX, USA) and following manufacturer instructions, blood serum samples were analyzed for concentrations of total proteins, albumin, urea-N, glucose, glutamate-pyruvate transaminase (GPT), glutamate-oxaloacetate transaminase (GOT), triglycerides, high-density lipoprotein cholesterol (HDL), low-density lipoprotein cholesterol (LDL), non-esterified fatty acids (NEFA) and beta-hydroxybutyric acid (BHBA) by Ultraviolet-Visible spectrophotometry. Globulin concentration was calculated by subtracting albumin values from their corresponding total protein values.

\subsection{Lactational Performance}

Does were hand milked twice daily at 09:00 and 21:00 h during the last two weeks of each experimental period. Both the morning and evening milk were mixed together and $10 \%$ of the recorded milk yield was taken daily and analyzed for different components (total solids, solids not fat, fat, protein, lactose and ash) using infrared spectrophotometry (Milkotester LM2, Belovo, Bulgaria).

Milk was analyzed for fatty acid proportions using methyl esters prepared by base-catalyzed methanolysis of the glycerides ( $\mathrm{NaOH}$ in methanol), following the standards of the International Dairy Federation. Samples were analyzed using an Agilent 19091J-413 HP-5 column containing 5\% phenyl methyl siloxane $(30 \mathrm{~m} \times 0.32 \mathrm{~mm}$ i.d., $\mathrm{df}=0.25 \mu \mathrm{m}$; Agilent Technologies Inc., Palo Alto, CA, USA) on a gas chromatography (Hewlett-Packard, Model 6890, Palo Alto, CA, USA) equipped with a flame ionization detector.

Milk gross energy, fat-corrected milk (FCM) and energy-corrected milk (ECM) were estimated according to Tyrrell and Reid [22], NRC [23] and Sjaunja et al. [24] equations, respectively.

\subsection{Statistical Analyses}

The Shapiro-Wilk test was used to test the normal distribution of variables. For the small number of variables that showed significance for the Shapiro-Wilk test, data transformation (e.g., natural log, inverse of the natural log, square root, and inverse of the square root) was applied before reanalyzing the normality of the residuals. Using a quintuplicate $3 \times 3$ Latin square design with three periods and three treatments, data were analyzed with PROC MIXED of SAS 9.4 (SAS Inst., Inc., Cary, NC, USA). Individual does were the experimental units. The statistical model was: $Y_{i j k l}=\mu+S_{i}+A_{j}+T_{k}+D_{l}\left(S_{i}\right)$ 
$+E_{i j k l}$, where $Y_{i j k l}$ is each individual observation for a given variable, $\mu$ is the overall mean, $S_{i}$ is the square effect, $A_{j}$ is the treatment effect, $T_{k}$ is the period effect, $D_{l}\left(S_{i}\right)$ is the effect of a doe within the square and $\mathrm{E}_{\mathrm{ijkl}}$ is the residual error. When $F$-test was significant at $p<0.05$, values of means were compared using the difference probability option of the least squares mean statement. Polynomial (linear and quadratic) contrasts were used to describe responses to increasing doses of N. oculata.

\section{Results}

\subsection{Growth, Milk Yield, Composition, Efficiency and Fatty Acid Profile}

For initial and final body weight as well as daily weight changes, no differences were observed with feeding NOM5 and NOM10 treatments (Table 3). Both of NOM5 and NOM10 treatments linearly improved $(p<0.01)$ daily milk production expressed as actual, ECM and FCM, as well as the yields of milk components $(p<0.05)$. Additionally, NOM5 and NOM10 enhanced the lactose concentration relative to the control. Linear increase in feed efficiency expressed as actual milk production/feed consumed $(p=0.019)$ or ECM/feed consumed $(p=0.035)$ were observed with NOM5 and NOM10 treatments (Table 3).

Table 3. Weight changes, milk production and composition of lactating Nubian goats fed a basal diet supplemented with $N$. oculata.

\begin{tabular}{|c|c|c|c|c|c|c|c|c|}
\hline & \multicolumn{3}{|c|}{ Treatments ${ }^{1}$} & \multirow{2}{*}{ SEM } & \multicolumn{4}{|c|}{$p$ Value } \\
\hline & Control & NOM5 & NOM10 & & Treatment & Period & Linear & Quadratic \\
\hline \multicolumn{9}{|c|}{ Body weight, $\mathrm{kg}$} \\
\hline Initial & 32.9 & 33.2 & 32.9 & 0.31 & 0.797 & 0.725 & 0.904 & 0.510 \\
\hline Final & 31.6 & 31.9 & 31.8 & 0.47 & 0.896 & 0.024 & 0.789 & 0.702 \\
\hline $\begin{array}{c}\text { Daily } \\
\text { changes, g/d }\end{array}$ & -16.0 & -14.9 & -13.2 & 5.55 & 0.938 & 0.063 & 0.725 & 0.959 \\
\hline \multicolumn{9}{|c|}{ Production, g/d } \\
\hline Milk & $1034^{\mathrm{b}}$ & $1144^{\mathrm{a}}$ & $1185^{\mathrm{a}}$ & 30.7 & 0.004 & 0.091 & 0.001 & 0.368 \\
\hline $\mathrm{ECM}$ & $973^{b}$ & $1073^{a}$ & $1110^{a}$ & 29.4 & 0.006 & 0.042 & 0.002 & 0.392 \\
\hline $\mathrm{FCM}(4 \%)$ & $989^{b}$ & $1088^{a}$ & $1118^{a}$ & 29.5 & 0.010 & 0.045 & 0.004 & 0.348 \\
\hline Total solids & $126^{\mathrm{b}}$ & $140^{\mathrm{a}}$ & $145^{\mathrm{a}}$ & 3.8 & 0.003 & 0.064 & 0.008 & 0.365 \\
\hline Solids not fat & $87.4^{\mathrm{b}}$ & $97.8^{a}$ & $102^{\mathrm{a}}$ & 2.7 & 0.001 & 0.095 & 0.003 & 0.384 \\
\hline Protein & 34.0 & 36.0 & 37.6 & 1.06 & 0.065 & 0.018 & 0.021 & 0.875 \\
\hline Fat & $38.3^{b}$ & $42.0^{\mathrm{a}}$ & $42.9^{\mathrm{a}}$ & 1.19 & 0.023 & 0.032 & 0.009 & 0.351 \\
\hline Lactose & $45.0^{\mathrm{b}}$ & $52.4^{\mathrm{a}}$ & $54.9^{\mathrm{a}}$ & 1.48 & $<0.001$ & 0.283 & $<0.001$ & 0.198 \\
\hline Ash & $8.45^{b}$ & $9.46^{\mathrm{a}}$ & $9.84^{\mathrm{a}}$ & 0.28 & 0.003 & 0.158 & 0.001 & 0.365 \\
\hline $\begin{array}{l}\text { Milk energy } \\
\text { content, } \\
\mathrm{MJ} / \mathrm{kg}\end{array}$ & $3.01^{b}$ & $3.33^{\mathrm{a}}$ & $3.45^{\mathrm{a}}$ & 0.091 & 0.005 & 0.046 & 0.002 & 0.362 \\
\hline \multicolumn{9}{|c|}{ Milk composition, $\mathrm{g} / \mathrm{kg}$} \\
\hline Total solids & 122 & 122 & 123 & 0.9 & 0.818 & 0.765 & 0.529 & 0.993 \\
\hline Solids not fat & 84.6 & 85.4 & 86.4 & 0.65 & 0.178 & 0.990 & 0.065 & 0.931 \\
\hline Protein & 32.9 & 31.4 & 31.7 & 0.41 & 0.308 & 0.186 & 0.059 & 0.075 \\
\hline Fat & 37.1 & 36.7 & 36.2 & 0.43 & 0.288 & 0.249 & 0.119 & 0.879 \\
\hline Lactose & $43.6^{b}$ & $45.8^{\mathrm{a}}$ & $46.4^{\mathrm{a}}$ & 0.45 & 0.003 & 0.280 & 0.001 & 0.157 \\
\hline Ash & 8.18 & 8.28 & 8.30 & 0.102 & 0.673 & 0.052 & 0.409 & 0.750 \\
\hline $\begin{array}{l}\text { Milk energy } \\
\text { output, MJ/d }\end{array}$ & 2.92 & 2.91 & 2.91 & 0.024 & 0.979 & 0.431 & 0.861 & 0.919 \\
\hline \multicolumn{9}{|c|}{ Milk efficiency } \\
\hline Milk/DMI & $0.98^{b}$ & $1.07^{\mathrm{a}}$ & $1.11^{\mathrm{a}}$ & 0.032 & 0.019 & 0.061 & 0.006 & 0.501 \\
\hline ECM/DMI & $0.92^{b}$ & $1.00^{\mathrm{a}}$ & $1.04^{\mathrm{a}}$ & 0.031 & 0.033 & 0.035 & 0.011 & 0.519 \\
\hline
\end{tabular}

Means with different superscripts within a row differ $(p<0.05)$. DMI, dry matter intake; ECM, energy corrected milk; FCM, fat corrected milk; SEM, standard error of the mean. ${ }^{1}$ Control diet comprising ( $/ \mathrm{kg}$ DM): $500 \mathrm{~g}$ of concentrates feed, $300 \mathrm{~g}$ berseem clover and $200 \mathrm{~g}$ wheat straw without supplements (Control treatment) or with $5 \mathrm{~g}$ (NOM5 treatment) or $10 \mathrm{~g}$ of $\mathrm{N}$. oculata/doe/d (NOM10 treatment) supplement. 
As shown in Table 4, without affecting milk individual fatty acids or total SFA concentrations, NOM5 and NOM10 treatments reduced $(p=0.002)$ C16:0 concentration and atherogenic index $(p=0.004)$ but improved $(p<0.01)$ of C20:5n3 (EPA), UFA and mono UFA concentrations in relation to the control treatment.

Table 4. Fatty acids profile (g/100 g total fatty acids) in milk of lactating Nubian goats fed a basal diet supplemented with N. oculata.

\begin{tabular}{|c|c|c|c|c|c|c|c|c|}
\hline & \multicolumn{3}{|c|}{ Treatments 1} & \multirow{2}{*}{ SEM } & \multicolumn{4}{|c|}{$p$ Value } \\
\hline & Control & NOM5 & NOM10 & & Treatment & Period & Linear & Quadratic \\
\hline C4:0 & 2.99 & 3.00 & 2.63 & 0.312 & 0.630 & 0.803 & 0.412 & 0.626 \\
\hline C6:0 & 2.11 & 1.94 & 2.02 & 0.133 & 0.681 & 0.040 & 0.632 & 0.468 \\
\hline C8:0 & 2.24 & 2.23 & 2.22 & 0.116 & 0.991 & 0.974 & 0.894 & 0.988 \\
\hline C10:0 & 5.24 & 5.18 & 5.14 & 0.174 & 0.915 & 0.475 & 0.679 & 0.965 \\
\hline C11:0 & 0.90 & 0.85 & 0.87 & 0.038 & 0.619 & 0.460 & 0.528 & 0.459 \\
\hline $\mathrm{C} 12: 0$ & 3.30 & 3.27 & 3.17 & 0.128 & 0.753 & 0.802 & 0.483 & 0.801 \\
\hline C14:0 & 9.38 & 9.30 & 8.84 & 0.210 & 0.173 & 0.494 & 0.083 & 0.487 \\
\hline C14:1 & 0.68 & 0.67 & 0.68 & 0.082 & 0.989 & 0.844 & 0.993 & 0.883 \\
\hline C15:0 & 0.55 & 0.53 & 0.56 & 0.035 & 0.875 & 0.353 & 0.965 & 0.610 \\
\hline $\mathrm{C} 16: 0$ & $26.2^{a}$ & $24.4^{\mathrm{b}}$ & $24.3^{b}$ & 0.38 & 0.002 & 0.253 & 0.002 & 0.089 \\
\hline $\mathrm{C} 16: 1$ & 1.13 & 1.28 & 1.19 & 0.109 & 0.617 & 0.617 & 0.674 & 0.379 \\
\hline C17:0 & 0.84 & 0.86 & 0.81 & 0.066 & 0.844 & 0.230 & 0.751 & 0.630 \\
\hline C18:0 & 16.3 & 16.7 & 16.3 & 0.30 & 0.631 & 0.551 & 0.971 & 0.343 \\
\hline C18:1 n9T & $23.8^{\mathrm{b}}$ & $25.2^{\mathrm{ab}}$ & $26.9^{a}$ & 0.58 & 0.004 & 0.632 & 0.009 & 0.861 \\
\hline C18:1 n9C & 2.61 & 2.82 & 2.58 & 0.151 & 0.496 & 0.496 & 0.922 & 0.243 \\
\hline $\begin{array}{c}\text { trans-10, } \\
\text { cis-12C18:2 }\end{array}$ & 0.27 & 0.29 & 0.30 & 0.015 & 0.474 & 0.562 & 0.227 & 0.975 \\
\hline $\begin{array}{c}\text { cis-9, } \\
\text { trans-11C18:2 }\end{array}$ & 0.17 & 0.18 & 0.19 & 0.011 & 0.598 & 0.250 & 0.340 & 0.751 \\
\hline C18:3 n-3 & 0.16 & 0.17 & 0.17 & 0.008 & 0.713 & 0.186 & 0.480 & 0.683 \\
\hline C18:3 n-6 & 0.38 & 0.39 & 0.40 & 0.016 & 0.616 & 0.046 & 0.337 & 0.867 \\
\hline C20:0 & 0.67 & 0.67 & 0.66 & 0.028 & 0.926 & 0.667 & 0.700 & 0.975 \\
\hline C20:5n-3 & $0.15^{c}$ & $0.17^{b}$ & $0.20^{a}$ & 0.009 & 0.002 & 0.132 & 0.006 & 0.510 \\
\hline$C 22: 5 n-3$ & 0.21 & 0.22 & 0.21 & 0.011 & 0.804 & 0.587 & 0.716 & 0.586 \\
\hline SFA & 70.8 & 68.9 & 67.5 & 1.58 & 0.303 & 0.650 & 0.116 & 0.775 \\
\hline UFA & $29.6^{b}$ & $31.4^{\mathrm{a}}$ & $32.9^{a}$ & 0.58 & 0.002 & 0.667 & 0.006 & 0.776 \\
\hline Mono UFA & $28.2^{b}$ & $30.0^{\mathrm{a}}$ & $31.4^{\mathrm{a}}$ & 0.57 & 0.003 & 0.629 & 0.007 & 0.777 \\
\hline Poly UFA & $1.35^{b}$ & $1.42^{\mathrm{ab}}$ & $1.48^{\mathrm{a}}$ & 0.035 & 0.049 & 0.368 & 0.015 & 0.908 \\
\hline Total CLA & 0.44 & 0.47 & 0.49 & 0.047 & 0.059 & 0.035 & 0.153 & 0.057 \\
\hline Omega-6/omega-3 & 2.32 & 2.28 & 2.34 & 0.101 & 0.896 & 0.515 & 0.847 & 0.673 \\
\hline UFA/SFA & $0.42^{b}$ & $0.46^{\mathrm{a}}$ & $0.49^{a}$ & 0.012 & 0.003 & 0.659 & 0.007 & 0.827 \\
\hline $\begin{array}{l}\text { Atherogenic } \\
\text { index }^{2}\end{array}$ & $2.28^{a}$ & $2.10^{b}$ & $1.92^{b}$ & 0.068 & 0.004 & 0.912 & 0.001 & 0.730 \\
\hline
\end{tabular}

Means with different superscripts within a row differ $(p<0.05)$. CLA, conjugated linoleic acid (trans-10, cis-12 C18:2 and cis-9, trans-11 C18:2); SEM, standard error of the mean; SFA, total saturated fatty acids; UFA, total unsaturated fatty acids. ${ }^{1}$ Control diet comprising ( $\left./ \mathrm{kg} \mathrm{DM}\right): 500 \mathrm{~g}$ of concentrates feed, $300 \mathrm{~g}$ berseem clover and $200 \mathrm{~g}$ wheat straw without supplement (Control treatment) or with $5 \mathrm{~g}$ (NOM5 treatment) or $10 \mathrm{~g}$ of N. oculata/doe/d (NOM10 treatment) supplement. ${ }^{2}$ Calculated according to Ulbricht and Southgate [25]: atherogenic index $=(\mathrm{C} 12: 0+$ $4 \times \mathrm{C} 14: 0+\mathrm{C} 16: 0) / \Sigma$ of UFA.

\subsection{Voluntary Intake and Nutrient Digestibility}

N. oculata supplementation had no effect on feed consumption (Table 5). Except for EE digestibility, NOM5 and NOM10 supplementations enhanced $(p<0.01)$ nutrient digestibility. Relative to the control treatment, NOM5 and NOM10 treatments showed higher $(p<0.001)$ digestible nutrients and energy value. 
Table 5. Intake and nutrient digestibility of basal diet supplemented with N. oculata.

\begin{tabular}{|c|c|c|c|c|c|c|c|c|}
\hline & \multicolumn{3}{|c|}{ Treatments ${ }^{1}$} & \multirow{2}{*}{ SEM } & \multicolumn{4}{|c|}{$p$ Value } \\
\hline & Control & NOM5 & NOM10 & & Treatment & Period & Linear & Quadratic \\
\hline Intake, $\mathrm{g} / \mathrm{d}$ & 1067 & 1075 & 1072 & 17.0 & 0.944 & 0.282 & 0.837 & 0.788 \\
\hline \multicolumn{9}{|c|}{ Digestibility, g/kg } \\
\hline Dry matter & $581^{b}$ & $623^{a}$ & $627^{a}$ & 5.3 & $<0.001$ & 0.019 & $<0.001$ & $<0.001$ \\
\hline Organic matter & $574^{b}$ & $608^{a}$ & $610^{\mathrm{a}}$ & 1.8 & $<0.001$ & $<0.001$ & $<0.001$ & $<0.001$ \\
\hline Crude protein & $579^{b}$ & $615^{a}$ & $621^{\mathrm{a}}$ & 5.8 & $<0.001$ & $<0.001$ & $<0.001$ & 0.043 \\
\hline Ether extract & 591 & 607 & 605 & 5.4 & 0.078 & $<0.001$ & 0.067 & 0.175 \\
\hline $\begin{array}{l}\text { Non-structural } \\
\text { carbohydrates }\end{array}$ & $549^{\mathrm{b}}$ & $603^{a}$ & $613^{a}$ & 5.7 & $<0.001$ & 0.152 & $<0.001$ & 0.003 \\
\hline Neutral detergent fiber & $555^{b}$ & $592^{\mathrm{a}}$ & $604^{\mathrm{a}}$ & 6.9 & $<0.001$ & 0.002 & $<0.001$ & 0.170 \\
\hline Acid detergent fiber & $542^{b}$ & $583^{a}$ & $584^{\mathrm{a}}$ & 7.5 & 0.003 & 0.151 & 0.004 & 0.039 \\
\hline Cellulose & $551^{\mathrm{b}}$ & $589^{\text {a }}$ & $597^{a}$ & 8.9 & 0.002 & 0.114 & 0.009 & 0.187 \\
\hline Hemicellulose & $548^{\mathrm{b}}$ & $592^{\mathrm{a}}$ & $587^{\mathrm{a}}$ & 8.3 & 0.001 & 0.126 & 0.002 & 0.023 \\
\hline \multicolumn{9}{|c|}{ Digestible nutrients and energy value ${ }^{2}$} \\
\hline $\begin{array}{l}\text { Digestible crude } \\
\text { protein, } \mathrm{g} / \mathrm{kg} \text { DM }\end{array}$ & $535^{b}$ & $572^{\mathrm{a}}$ & $575^{\mathrm{a}}$ & 4.0 & $<0.001$ & 0.448 & $<0.001$ & 0.009 \\
\hline $\begin{array}{l}\text { Digestible crude } \\
\text { protein, } \mathrm{g} / \mathrm{kg} \mathrm{DM}\end{array}$ & $73.8^{b}$ & $78.4^{a}$ & $79.2^{\mathrm{a}}$ & 0.74 & $<0.001$ & $<0.001$ & $<0.001$ & 0.043 \\
\hline $\begin{array}{l}\text { Digestible energy, } \\
\mathrm{MJ} / \mathrm{kg} \mathrm{DM}\end{array}$ & $2.36^{b}$ & $2.52^{a}$ & $2.54^{\mathrm{a}}$ & 0.017 & $<0.001$ & 0.484 & $<0.001$ & 0.008 \\
\hline $\begin{array}{c}\text { Metabolizable energy, } \\
\text { MJ/kg DM }\end{array}$ & $2.38^{\mathrm{b}}$ & $2.55^{a}$ & $2.56^{a}$ & 0.018 & $<0.001$ & 0.457 & $<0.001$ & 0.009 \\
\hline $\begin{array}{c}\text { Net energy for lactation, } \\
\mathrm{MJ} / \mathrm{kg} \mathrm{DM}\end{array}$ & $1.19^{\mathrm{b}}$ & $1.28^{a}$ & $1.29^{\mathrm{a}}$ & 0.010 & $<0.001$ & 0.419 & $<0.001$ & 0.001 \\
\hline
\end{tabular}

Means with different superscripts within a row differ $(p<0.05)$. SEM, standard error of the mean. ${ }^{1}$ Control diet comprising ( $/ \mathrm{kg} \mathrm{DM}): 500 \mathrm{~g}$ of concentrates feed, $300 \mathrm{~g}$ berseem clover and $200 \mathrm{~g}$ wheat straw without supplement (Control treatment) or with $5 \mathrm{~g}$ (NOM5 treatment) or $10 \mathrm{~g}$ of $\mathrm{N}$. oculata/doe/d (NOM10 treatment) supplement.

2 Calculated according to NRC [23].

\subsection{Serum Metabolites and Rumen Fermentation}

Treatments had no effect serum total protein, albumin, globulin and urea-N (Table 6). Moreover, NOM5 and NOM10 had no effect on serum GOT and GPT, triglycerides, LDL, NEFA, HDL or BHBA concentrations; however, they increased glucose concentration.

Table 6. Serum metabolites (g/dL, unless stated otherwise) of lactating Nubian goats fed a basal diet supplemented with $N$. oculata.

\begin{tabular}{|c|c|c|c|c|c|c|c|c|}
\hline & \multicolumn{3}{|c|}{ Treatments ${ }^{1}$} & \multirow{2}{*}{ SEM } & \multicolumn{4}{|c|}{$p$ Value } \\
\hline & Control & NOM5 & NOM10 & & Treatment & Period & Linear & Quadratic \\
\hline Total proteins & 6.59 & 6.55 & 6.64 & 0.059 & 0.543 & 0.112 & 0.570 & 0.345 \\
\hline Albumin & 3.43 & 3.47 & 3.46 & 0.035 & 0.713 & 0.391 & 0.597 & 0.531 \\
\hline Globulin & 3.16 & 3.08 & 3.18 & 0.056 & 0.360 & 0.015 & 0.787 & 0.163 \\
\hline Albumin/globulin ratio & 1.09 & 1.13 & 1.09 & 0.024 & 0.372 & 0.014 & 0.921 & 0.163 \\
\hline Urea-N & 39.4 & 39.5 & 39.6 & 0.85 & 0.986 & 0.002 & 0.869 & 0.991 \\
\hline Glucose & $61.9^{b}$ & $68.5^{\mathrm{a}}$ & $68.2^{\mathrm{a}}$ & 0.75 & $<0.001$ & 0.188 & $<0.001$ & 0.005 \\
\hline GPT, Units/L & 15.6 & 15.4 & 15.5 & 0.27 & 0.843 & 0.977 & 0.776 & 0.612 \\
\hline GOT, Units/L & 31.2 & 31.7 & 31.2 & 0.62 & 0.786 & 0.133 & 0.938 & 0.493 \\
\hline Triglycerides & 140 & 137 & 134 & 1.9 & 0.093 & 0.546 & 0.030 & 0.919 \\
\hline HDL & 50.4 & 49.7 & 50.1 & 1.01 & 0.897 & 0.758 & 0.817 & 0.688 \\
\hline LDL & 53.7 & 51.8 & 51.4 & 1.60 & 0.570 & 0.918 & 0.323 & 0.711 \\
\hline NEFA & 0.95 & 1.04 & 1.02 & 0.030 & 0.139 & 0.278 & 0.161 & 0.155 \\
\hline BHBA & 1.68 & 1.63 & 1.63 & 0.042 & 0.647 & 0.169 & 0.415 & 0.655 \\
\hline
\end{tabular}

Means with different superscripts within a row differ $(p<0.05)$. SEM, standard error of the mean. BHBA, Beta-hydroxybutyric acid; GOT, glutamate-oxaloacetate transaminase; GPT, glutamate-pyruvate transaminase; HDL, High-density lipoprotein cholesterol; LDL, Low-density lipoprotein cholesterol; NEFA, Non-esterified fatty acids. ${ }^{1}$ Control diet comprising ( $\left./ \mathrm{kg} \mathrm{DM}\right): 500 \mathrm{~g}$ of concentrates feed, $300 \mathrm{~g}$ berseem clover and $200 \mathrm{~g}$ wheat straw without supplement (Control treatment) or with $5 \mathrm{~g}$ (NOM5 treatment) or $10 \mathrm{~g}$ of $\mathrm{N}$. oculata/doe/d (NOM10 treatment) supplement. 
Dietary treatments had no effect ruminal $\mathrm{pH}$ or ammonia-N (Table 7). N. oculata linearly increased total VFA $(p=0.002)$ and propionic acid $(p=0.022)$ without affecting ruminal acetic and butyric acid proportions. Moreover, the NOM10 treatment linearly decreased $(p=0.045)$ calculated $\mathrm{CH}_{4}$ production.

Table 7. Ruminal fermentation in lactating Nubian goats fed a basal diet supplemented with N. oculata.

\begin{tabular}{|c|c|c|c|c|c|c|c|c|}
\hline & \multicolumn{3}{|c|}{ Treatments $^{1}$} & \multirow{2}{*}{ SEM } & \multicolumn{4}{|c|}{$p$ Value } \\
\hline & Control & NOM5 & NOM10 & & Treatment & Period & Linear & Quadratic \\
\hline $\mathrm{pH}$ & 5.94 & 6.03 & 6.14 & 0.060 & 0.078 & 0.077 & 0.025 & 0.904 \\
\hline Ammonia-N, g/L & 25.3 & 26.3 & 27.3 & 0.79 & 0.239 & 0.357 & 0.093 & 0.973 \\
\hline Volatile fatty acids, $\mathrm{mmol} / \mathrm{L}$ & $115^{\mathrm{c}}$ & $124^{\mathrm{b}}$ & $133^{\mathrm{a}}$ & 2.700 & 0.002 & $<0.001$ & $<0.001$ & 0.901 \\
\hline Acetic, $\mathrm{mmol} / 100 \mathrm{mmol}$ & 56.5 & 57.4 & 55.1 & 1.63 & 0.617 & 0.799 & 0.565 & 0.430 \\
\hline Propionic, $\mathrm{mmol} / 100 \mathrm{mmol}$ & $25.0^{\mathrm{b}}$ & $27.4^{\mathrm{a}}$ & $27.8^{\mathrm{a}}$ & 0.850 & 0.045 & 0.468 & 0.031 & 0.356 \\
\hline Butyric, $\mathrm{mmol} / 100 \mathrm{mmol}$ & 16.4 & 13.5 & 13.5 & 1.29 & 0.201 & 0.381 & 0.120 & 0.372 \\
\hline Acetic/propionic ratio & 2.31 & 2.11 & 2.00 & 0.123 & 0.223 & 0.413 & 0.090 & 0.799 \\
\hline Methane production ${ }^{2}$ & $25.1^{\mathrm{a}}$ & $23.7^{\mathrm{ab}}$ & $22.5^{b}$ & 0.670 & 0.045 & 0.943 & 0.014 & 0.882 \\
\hline
\end{tabular}

Means with different superscripts within a row differ $(p<0.05)$. SEM, standard error of the mean. ${ }^{1}$ Control diet comprising $(/ \mathrm{kg} \mathrm{DM}): 500 \mathrm{~g}$ of concentrates mixture, $300 \mathrm{~g}$ berseem clover and $200 \mathrm{~g}$ wheat straw without supplement (Control treatment) or with $5 \mathrm{~g}$ (NOM5 treatment) or $10 \mathrm{~g}$ of $\mathrm{N}$. oculata/doe/d (NOM10 treatment) supplement. ${ }^{2}$ Methane production $(\mathrm{mmol} / \mathrm{L})=0.45$ (Acetic) -0.275 (Propionic) +0.4 (Butyric) [26]

\section{Discussion}

\subsection{Lactational Performance}

The NOM5 and NOM10 linearly increased daily milk yield for the actual (by about 10.6 and 1.6\%), ECM (by 10.2 and14.1\%) and FCM (by 10 and 13.1\%) respectively. Increased milk yield with unaffected feed consumption results in enhanced feed (milk) efficiency by about expressed as actual milk production/feed intake (by 9.5 and 13.4\%, respectively) or ECM/feed intake (by 9.1 and $12.8 \%$, respectively). Enhanced nutrient digestion and ruminal fermentation are majorly responsible for the enhanced milk yield [27]. As it was discussed later, increased lactose content with N. oculata may also be responsible for the improved milk yield [28].

N. oculata increase the concentration of lactose (by 5 and 6.3\% for NOM5 and NOM10 treatments respectively) as a result of increased propionic acid production. Propionic acid, the precursor for gluconeogenesis and lactose synthesis, favorably affect milk production [28]. The minimal effect of N. oculata on milk fat concentration is in line with the result of milk fat precursors in rumen, namely acetate and butyrate as well as precursors in blood namely NEFA and BHBA [29,30]. Lamminen et al. [3] reported higher milk fat with microalgae supplementation. The level of supplementation may be responsible about the inconsistency. Póti et al. [31] observed that feeding microalga as fat supplement to Hungarian native goat increased milk fat content without affecting other milk components.

\subsection{Milk Fatty Acid Profile}

Minor changes in milk fatty acid profile were expected since microalgae supplementation did not affect fat concentration in milk; however, some changes were noted. Composition of milk fatty acid is influenced by the type of fatty acids consumed by animal. Generally, dietary factors and absorbed fats affect milk fatty acid profile [32]. With exception of C16:0 and C18:1 n9T, feeding N. oculata had no effect on individual fatty acids. In their review, Altomonte et al. [33] summarized that greatest changes in the profile of milk fatty acids were related to increases in long-chain PUFA and omega-3 fatty acids and to accompanying decreases in SFA.

N. oculata decreased and increased C16:0 and C18:1 concentrations respectively, indicating enhanced milk nutritive value for human health [34]. Vahmani et al. [17] observed a reduced C16:0 concentration by $12 \%$ with PUFA-rich microalgae to lactating cows. This may be due to the suppression of mammary de novo 
fatty acids synthesis [35]. Moreover, increased C18:1 fatty acid relates to increased mammary supply of C18:0 with microalgae supplementation, providing more substrate for mammary $\Delta^{9}$-desaturase.

N. oculata fed goats produced EPA fatty acid-fortified milk (increases of 12.8 and $36.1 \%$ respectively for NOM5 and NOM10 treatments), without affecting DHA concentration. This may be a result of the inclusion of EPA-rich microalgae in the diet [3]. Efficiencies of transfer of both of EPA and DHA from diet to milk have been estimated to be 2.6 and $4.1 \%$, respectively [36] and can be increased several fold when animals' diet is supplemented with sources protected from ruminal biohydrogenation [37,38]. Lamminen et al. [3] reported increased EPA concentration fourfold with $N$. oculata feeding to lactating cows. Moreover, Vahmani et al. [17] observed dietary supplementation of lactating cows with EPA-rich supplementation improved milk fat concentrations of EPA by $100 \%$ and DHA by $86 \%$.

Theoretically, majority of consumed poly UFA undergo ruminal biohydrogenation and are thus not incorporated into milk unaltered; however, protecting them from ruminal biohydrogenation increases their excretion in milk. N. oculata increased UFA by 6.3 and $11.2 \%$, respectively for NOM5 and NOM10 treatments, resulting in production healthier milk for human consumption. Vahmani et al. [17] observed a decreased SFA by $9 \%$ and increased C18:1 n9T concentration by $81 \%$ with UFA-rich microalgae. Boeckaert et al. [39] reported that UFA-rich microalgae suppress in vitro biohydrogenation of fatty acids, resulting in reduced SFA and increased UFA.

The gradual increases in C20:5n-3 ( $\alpha$-linolenic acid) proportions with increasing N. oculata indicates improve nutritive value of produced milk. A raise in EPA has been reported in cows supplemented with full-fatted microalgae biomass [40]. This result indicates that microalgae biomass may play a positive role in enhancing the health-promoting n-3 fatty acids in milk.

N. oculata decreased the concentrations of atherogenic index by 9.3 and $16.1 \%$ respectively for NOM5 and NOM10 treatments, buttressing the fact that feeding microalgae produced healthy milk. With the supplementation of rich fat microalga to Hungarian native goat, Póti et al. [31] noted increases in the concentrations of C4:0, C18:1t, C18:2, C18:3, C20:3, C20:5, C22:6, mon UFA, poly UFA, and n-3 fatty acids and decreases in the concentrations of C20:4 and SFA in milk. Additionally, they [31] observed that increasing the supply of n-3 poly UFA decreased the ratios of n-6/n-3 fatty acids and the atherogenic index.

\subsection{Intake and Digestibility}

Unexpectedly, N. oculata had no effect on feed consumption indicating unaffected palatability of the diets by the microalgae supplementation. Glover et al. [16] noted that feed consumption was not affected with feeding of ruminally-protected microalgae rich in DHA fatty acids. Feeding large quantities of microalgae causes palatability problems in ruminants as a result odor and taste of the microalgae [3] due to the 'grassy, vegetable, cucumber' flavors [41]. Lamminen et al. [3] observed minimal effects with feeding microalgae (Spirulina, Chlorella and Nannochloropsis) in the concentrate portion of a diet to lactating cows; however, they noted increased silage intake and decreased concentrate intake. The level of microalgae supplementation may be a reason for the inconsistency between experiments. In the present experiment, microalgae supplementation ranged between 0.49 to $0.94 \%$ compared with about 10-fold supplementation in Lamminen et al. [3].

N. oculata, at the two levels evaluated in the present experiment, enhanced nutrient digestibility without affecting the digestibility of $\mathrm{EE}$, revealing enhanced ruminal fermentation with the microalgae supplementation. The microalgae contains about $67 \%$ UFA of its content of total fatty acids which was expected to negatively affect fiber digestion [42,43] due to the adverse effect of UFA on the rumen microbiome [42,43]. These results reveal that the two rates/doses of microalgae in the current assay fell within tolerable range, as previously noted with feed intake. Additionally, result of digestibility confirms the result of Alves et al. [11] who reported that the UFA in N. oculata are in a protected form, which means that minimal ruminal biohydrogenation occurs to microalgae UFA and prevents their negative effects on ruminal microbiota. Otherwise, the increased nutrient digestibility indicates improved rumen microflora population/activity with the supplementation. Supplementation with 
UFA decreases the number of protozoa which causes increased total bacterial population as no or reduced predation of bacteria by protozoa occurs [6]. This result concurs with those of Gomaa et al. [6] who observed enhanced DM and NDF digestibility with N. oculata and sunflower oil mixture due to improved ruminal microbes' activity.

\subsection{Serum Metabolites}

All serum parameters in the present study were within the recommended ranges for healthy animals [44]. N. oculata supplementation did not affect blood total protein, protein fractions and urea-N, indicating marginal effects on the nutritional status and muscles protein catabolism [44]. Moreover, these results suggest normal activity. Serum urea-N is a good index of the kidney glomerular filtration [45]. Moreover, N. oculata did not affect the concentrations of serum GOT and GPT indicating minimal effects on liver function and reflects a high safety of feeding N. oculata to goats.

N. oculata did not affect the concentrations of serum triglycerides, LDL and HDL, indicating unaffected release of triglyceride-rich lipoproteins into the lymphatic system. Additionally, N. oculata did not affect the concentrations of serum NEFA and BHBA, revealing positive effects on minimizing body fat break down in goats supplemented with $N$. oculata. Such results suggest absence of a negative energy balance in the goats. Moreover, this indicates enhanced energy status of goats with N. oculata supplementation. These results are in line with the results of unaffected daily body weight loss.

N. oculata increased serum glucose concentration with 10.8 and $12.2 \%$, for NOM5 and NOM10 treatments, respectively which is the consequence of improved OM digestibility, total VFA concentration and propionic acid production, because about $73 \%$ of hepatic glucose production in ruminants is from ruminal propionic acid [46]. Interestingly, serum glucose results were paralleled with those of milk production, confirming the previously noted positive correlation between serum glucose and milk production in animals fed UFA supplemented diet [47].

\subsection{Ruminal Fermentation}

Values of ruminal $\mathrm{pH}$ [48] and concentrations of ruminal $\mathrm{NH}_{3}-\mathrm{N}$ [49] fell within the accepted ranges required for optimal ruminal microflora growth and activity, with no effect due to N. oculata feeding. Ruminal $\mathrm{NH}_{3}-\mathrm{N}$ concentration is an indirect evidence of unaffected duodenal flow of microbial $\mathrm{N}$ and efficiency [43]. That ruminal $\mathrm{NH}_{3}-\mathrm{N}$ was not affected, and digestibility of $\mathrm{CP}$ was improved with $N$. oculata feeding is an evidence of increased ruminal microbial synthesis and activity due to use of ruminal $\mathrm{NH}_{3}-\mathrm{N}$ and conversion of $\mathrm{N}$ into microbial protein. Kholif et al. [50] observed no alterations in rumen ammonia-N with UFA supplementation to lactating goats.

N. oculata improved ruminal total VFA concentration (by 8.1 and 15.5\% for NOM5 and NOM10 treatments respectively), without affecting ruminal $\mathrm{pH}$ possibly due to the enhanced OM and fibers digestibilities [51]. Feed digestion and activity of ruminal microflora determine the concentration of ruminal VFA [52]. Kholif et al. [50] showed that supplementing lactating goats with ruminally protected UFA (i.e., flaxseed seeds) increased ruminal VFA concentration through the reduction of ruminal protozoa, and increasing the dietary energy density with supplementation [53].

$N$. oculata increased the ruminal propionic acid proportions by 9.4 and $11.1 \%$ respectively for NOM5 and NOM10 treatments, reflecting a higher conversion of glycerol into propionic acid [49]; glycerol is the consequence of the breakdown of dietary fatty acids. Increased propionate concentration is due to improved OM and NSC digestibility. Moreover, it may also due to diversion of excess decreased NADH to propionic acid production as a result of improved accumulation of $\mathrm{H}$ due to inhibition of ruminal methanogens [54].

N. oculata did not affect ruminal acetic and butyric acids proportions, which is another evidence that the amounts of UFA from the microalgae in the present experiment were adequate to improve the goats' productive performance with no effect on ruminal cellulolytic bacterial activity.

N. oculata at high level (i.e., NOM10 treatment) decreased calculated $\mathrm{CH}_{4}$ production by $10.1 \%$. Wild et al. [55] reported that N. oculata decreased ruminal $\mathrm{CH}_{4}$ production in vitro. Additionally, 
Boeckaert et al. [56] and Fievez et al. [57] observed a declined ruminal methanogenesis in vitro. The presence of EPA and DHA fatty is the main reason [55-57]. Wild et al. [55] reported a negative relationship between $\mathrm{CH}_{4}$ and the EPA concentration $(\mathrm{r}=-0.51)$. However we did not measure ruminal microbiota, assumption that the presence of UFA in the algae can be toxic to ruminal protozoa, which is one of $\mathrm{CH}_{4}$ producers [58]. Protozoa are the main engulfers of rumen bacteria cells (20,000 cells $\left./ \mathrm{h}\right)$ [59]. Boeckaert et al. [39] reported that microalgae rich in UFA decreased the number of Isotricha prostoma and Isotricha intestinalis and some species of Epidinium caudatum ciliates.

\section{Conclusions}

Supplementation lactating Nubian goats' diet with N. oculata at 5 or $10 \mathrm{~g} /$ doe daily enhanced daily milk production and profile of milk fatty acids as increasing UFA and C20:5n-3 ( $\alpha$-linolenic acid) and decreasing SFA concentrations. Moreover, $N$. oculata enhanced nutrient digestion, and ruminal fermentation without affecting blood chemistry. The $5 \mathrm{~g} / \mathrm{d}$ of $N$. oculata microalgae is suggested for its using in lactating Nubian goats due to the cost.

Author Contributions: Conceptualization, A.E.K. and G.A.G.; methodology, A.E.K. and G.A.G.; software, A.E.K. and G.A.G.; validation, A.E.K., H.A.H. and G.A.G.; formal analysis, A.E.K. and G.A.G.; investigation, A.E.K. and G.A.G.; H.A.H.; data curation, A.E.K., H.A.H.; writing—original draft preparation, A.E.K.; writing-review and editing, A.E.K.; visualization, A.E.K., H.A.H.; supervision, H.A.H.; project administration, A.E.K. and G.A.G.; All authors have read and agreed to the published version of the manuscript.

Funding: This research received no external funding.

Conflicts of Interest: No conflict of interest.

\section{References}

1. Chisti, Y. Biodiesel from microalgae. Biotechnol. Adv. 2007, 25, 294-306. [CrossRef] [PubMed]

2. Efroymson, R.A.; Dale, V.H.; Langholtz, M.H. Socioeconomic indicators for sustainable design and commercial development of algal biofuel systems. GCB Bioenergy 2017, 9, 1005-1023. [CrossRef]

3. Lamminen, M.; Halmemies-Beauchet-Filleau, A.; Kokkonen, T.; Jaakkola, S.; Vanhatalo, A. Different microalgae species as a substitutive protein feed for soya bean meal in grass silage based dairy cow diets. Anim. Feed Sci. Technol. 2019, 247, 112-126. [CrossRef]

4. Peng, K.; Li, J.; Jiao, K.; Zeng, X.; Lin, L.; Pan, S.; Danquah, M.K. The Bioeconomy of Microalgal Biofuels. In Green Energy and Technology; Jacob-Lopes, E., Queiroz Zepka, L., Queiroz, M.I., Eds.; Springer International Publishing: Cham, Switzerland, 2018; pp. 157-169.

5. Kholif, A.E.; Hamdon, H.A.; Kassab, A.Y.; Farahat, E.S.A.; Azzaz, H.H.; Matloup, O.H.; Mohamed, A.G.; Anele, U.Y. Chlorella vulgaris microalgae and/or copper supplementation enhanced feed intake, nutrient digestibility, ruminal fermentation, blood metabolites and lactational performance of Boer goat. J. Anim. Physiol. Anim. Nutr. 2020, 104, 1595-1605. [CrossRef]

6. Gomaa, A.S.; Kholif, A.E.; Kholif, A.M.; Salama, R.; El-Alamy, H.A.; Olafadehan, O.A. Sunflower oil and Nannochloropsis oculata microalgae as sources of unsaturated fatty acids for mitigation of methane production and enhancing diets' nutritive value. J. Agric. Food Chem. 2018, 66, 1751-1759. [CrossRef]

7. Kholif, A.E.; Morsy, T.A.; Matloup, O.H.; Anele, U.Y.; Mohamed, A.G.; El-Sayed, A.B. Dietary Chlorella vulgaris microalgae improves feed utilization, milk production and concentrations of conjugated linoleic acids in the milk of Damascus goats. J. Agric. Sci. 2017, 155, 508-518. [CrossRef]

8. Kholif, A.E.; Olafadehan, O.A. Chlorella vulgaris microalgae in ruminant nutrition: A review of the chemical composition and nutritive value. Ann. Anim. Sci. 2021, in press.

9. Kholif, A.E.; Elghandour, M.M.Y.; Salem, A.Z.M.; Barbabosa, A.; Márquez, O.; Odongo, N.E. The effects of three total mixed rations with different concentrate to maize silage ratios and different levels of microalgae Chlorella vulgaris on in vitro total gas, methane and carbon dioxide production. J. Agric. Sci. 2017, 155, 494-507. [CrossRef]

10. Wild, K.J.; Steingaß, H.; Rodehutscord, M. Variability in nutrient composition and in vitro crude protein digestibility of 16 microalgae products. J. Anim. Physiol. Anim. Nutr. 2018, 102, 1306-1319. [CrossRef] 
11. Alves, S.P.; Mendonça, S.H.; Silva, J.L.; Bessa, R.J.B. Nannochloropsis oceanica, a novel natural source of rumen-protected eicosapentaenoic acid (EPA) for ruminants. Sci. Rep. 2018, 8. [CrossRef]

12. Kholif, A.E.; Kassab, A.Y.; Hamdon, H.A. Chlorella vulgaris microalgae and copper mixture supplementation enhanced the nutrient digestibility and milk attributes in lactating boer goats. Ann. Anim. Sci. 2021, in press. [CrossRef]

13. Archibeque, S.L.; Ettinger, A.; Willson, B.D. Nannochloropsis oculata as a source for animal feed. Acta Agron. Hungarica 2009, 57, 245-248. [CrossRef]

14. Durmic, Z.; Moate, P.J.; Eckard, R.; Revell, D.K.; Williams, R.; Vercoe, P.E. In vitro screening of selected feed additives, plant essential oils and plant extracts for rumen methane mitigation. J. Sci. Food Agric. 2014, 94, 1191-1196. [CrossRef] [PubMed]

15. Lee, J.Y.; Hwang, D.H. Dietary fatty acids and eicosanoids. In Fatty Acids in Foods and their Health Implications, 3rd Ed.; Chow, C.K., Ed.; CRC Press: Boca Raton, FL, USA, 2007; pp. 713-726. ISBN 9781420006902.

16. Glover, K.E.; Budge, S.; Rose, M.; Rupasinghe, H.P.V.; MacLaren, L.; Green-Johnson, J.; Fredeen, A.H. Effect of feeding fresh forage and marine algae on the fatty acid composition and oxidation of milk and butter. J. Dairy Sci. 2012, 95, 2797-2809. [CrossRef]

17. Vahmani, P.; Fredeen, A.H.; Glover, K.E. Effect of supplementation with fish oil or microalgae on fatty acid composition of milk from cows managed in confinement or pasture systems. J. Dairy Sci. 2013, 96, 6660-6670. [CrossRef]

18. Vizzarri, F. Book Review: Mason's World Encyclopedia of Livestock Breeds and Breeding; CABI: Boston, MA, USA, 2016; Volume 9, ISBN 9781780647944.

19. NRC. Nutrient Requirements for Sheep, Goats, Cervids, and New World Camelids; National Academy Press: Washington, DC, USA, 2007.

20. AOAC. Official Methods of Analysis, 16th ed.; Association of Official Analytical Chemists: Washington, DC, USA, 1980; Volume 52.

21. Stanier, R.Y.; Kunisawa, R.; Mandel, M.; Cohen-Bazire, G. Purification and properties of unicellular blue-green algae (order Chroococcales). Bacteriol. Rev. 1971, 35, 171-205. [CrossRef]

22. Tyrrell, H.F.; Reid, J.T. Prediction of the energy value of cow's milk. J. Dairy Sci. 1965, 48, 1215-1223. [CrossRef]

23. NRC. Nutrient Requirements of Dairy Cattle; National Academies Press: Washington, DC, USA, 2001; ISBN 978-0-309-06997-7.

24. Sjaunja, L.O.; Baevre, L.; Junkkarinen, L.; Pedersen, J.; Setala, J. A Nordic proposal for an energy corrected Milk (ECM) formula EAAP Publication 50: Performance Recording of Animals. EAAP Publ. 1991, 50, 156-157.

25. Ulbricht, T.L.V.; Southgate, D.A.T. Coronary heart disease: Seven dietary factors. Lancet 1991, 338, $985-992$. [CrossRef]

26. Moss, A.R.; Jouany, J.P.; Newbold, J. Methane production by ruminants: Its contribution to global warming. Anim. Res. 2000, 49, 231-253. [CrossRef]

27. Kholif, A.E.; Abdo, M.M.; Anele, U.Y.; El-Sayed, M.M.; Morsy, T.A. Saccharomyces cerevisiae does not work synergistically with exogenous enzymes to enhance feed utilization, ruminal fermentation and lactational performance of Nubian goats. Livest. Sci. 2017, 206, 17-23. [CrossRef]

28. Rigout, S.; Hurtaud, C.; Lemoscjuet, S.; Bach, A.; Rulquin, H. Lactational effect of propionic acid and duodenal glucose in cows. J. Dairy Sci. 2003, 86, 243-253. [CrossRef]

29. Matloup, O.H.; Abd El Tawab, A.M.; Hassan, A.A.; Hadhoud, F.I.; Khattab, M.S.A.; Khalel, M.S.; Sallam, S.M.A.; Kholif, A.E. Performance of lactating Friesian cows fed a diet supplemented with coriander oil: Feed intake, nutrient digestibility, ruminal fermentation, blood chemistry, and milk production. Anim. Feed Sci. Technol. 2017, 226, 88-97. [CrossRef]

30. Chilliard, Y.; Ferlay, A.; Mansbridge, R.M.; Doreau, M. Ruminant milk fat plasticity: Nutritional control of saturated, polyunsaturated, trans and conjugated fatty acids. Anim. Res. 2000, 49, 181-205. [CrossRef]

31. Póti, P.; Pajor, F.; Bodnár, Á.; Penksza, K.; Köles, P. Effect of micro-alga supplementation on goat and cow milk fatty acid composition. Chil. J. Agric. Res. 2015, 75, 259-263. [CrossRef]

32. Nafikov, R.A.; Soyeurt, H.; Beitz, D.C. Genetics of fatty acid composition in bovine milk and beef. In The Genetics of Cattle; Garrick, D.G., Ruvinsky, A., Eds.; CABI: Boston, MA, USA, 2014; pp. 433-450.

33. Altomonte, I.; Salari, F.; Licitra, R.; Martini, M. Use of microalgae in ruminant nutrition and implications on milk quality-A review. Livest. Sci. 2018, 214, 25-35. [CrossRef] 
34. Simopoulos, A.P. The importance of the omega-6/omega-3 fatty acid ratio in cardiovascular disease and other chronic diseases. Exp. Biol. Med. 2008, 233, 674-688. [CrossRef]

35. Ahnadi, C.E.; Beswick, N.; Delbecchi, L.; Kennelly, J.J.; Lacasse, P. Addition of fish oil to diets for dairy cows. II. Effects on milk fat and gene expression of mammary lipogenic enzymes. J. Dairy Res. 2002, 69, 521-531. [CrossRef]

36. Chilliard, Y.; Ferlay, A.; Doreau, M. Effect of different types of forages, animal fat or marine oils in cow's diet on milk fat secretion and composition, especially conjugated linoleic acid (CLA) and polyunsaturated fatty acids. Livest. Prod. Sci. 2001, 70, 31-48. [CrossRef]

37. Franklin, S.T.; Martin, K.R.; Baer, R.J.; Schingoethe, D.J.; Hippen, A.R. Dietary marine algae (Schizochytrium sp.) increases concentrations of conjugated linoleic, docosahexaenoic and transvaccenic acids in milk of dairy cows. J. Nutr. 1999, 129, 2048-2052. [CrossRef]

38. Kitessa, S.M.; Gulati, S.K.; Simos, G.C.; Ashes, J.R.; Scott, T.W.; Fleck, E.; Wynn, P.C. Supplementation of grazing dairy cows with rumen-protected tuna oil enriches milk fat with n-3 fatty acids without affecting milk production or sensory characteristics. Br. J. Nutr. 2004, 91, 271-277. [CrossRef] [PubMed]

39. Boeckaert, C.; Vlaeminck, B.; Mestdagh, J.; Fievez, V. In vitro examination of DHA-edible micro algae. 1. Effect on rumen lipolysis and biohydrogenation of linoleic and linolenic acids. Anim. Feed Sci. Technol. 2007, 136, 63-79. [CrossRef]

40. Craddock, J.C.; Neale, E.P.; Probst, Y.C.; Peoples, G.E. Algal supplementation of vegetarian eating patterns improves plasma and serum docosahexaenoic acid concentrations and omega-3 indices: A systematic literature review. J. Hum. Nutr. Diet. 2017, 30, 693-699. [CrossRef] [PubMed]

41. Van Durme, J.; Goiris, K.; De Winne, A.; De Cooman, L.; Muylaert, K. Evaluation of the volatile composition and sensory properties of five species of microalgae. J. Agric. Food Chem. 2013, 61, 10881-10890. [CrossRef]

42. Doreau, M.; Chilliard, Y. Effects of ruminal or postruminal fish oil supplementation on intake and digestion in dairy cows. Reprod. Nutr. Dev. 1997, 37, 113-124. [CrossRef]

43. Kucuk, O.; Hess, B.W.; Rule, D.C. Soybean oil supplementation of a high-concentrate diet does not affect site and extent of organic matter, starch, neutral detergent fiber, or nitrogen digestion, but influences both ruminal metabolism and intestinal flow of fatty acids in limit-fed lambs. J. Anim. Sci. 2004, 82, 2985-2994. [CrossRef]

44. Etim, N.N.; Enyenihi, G.E.; Williams, M.E.; Udo, M.D.; Offiong, E.E.A. Haematological parameters: Indicators of the physiological status of farm animals. Br. J. Sci. 2013, 10, 33-45.

45. Hosten, A.O. BUN and Creatinine. In Clinical Methods: The History, Physical, and Laboratory Examinations; Walker, H.K., Hall, W.D., Hurst, J.W., Eds.; Butterworths: Boston, MA, USA, 1990; pp. 874-878. ISBN 040990077X.

46. Oh, Y.K.; Eun, J.S.; Lee, S.C.; Chu, G.M.; Lee, S.S.; Moon, Y.H. Responses of blood glucose, insulin, glucagon, and fatty acids to intraruminal infusion of propionate in Hanwoo. Asian-Australas. J. Anim. Sci. 2015, 28, 200-206. [CrossRef]

47. Kholif, A.E.; Morsy, T.A.; Abd El Tawab, A.M.; Anele, U.Y.; Galyean, M.L. Effect of Supplementing diets of Anglo-Nubian goats with soybean and flaxseed oils on lactational performance. J. Agric. Food Chem. 2016, 64, 6163-6170. [CrossRef]

48. Ryle, M.; Ørskov, E.R. Energy Nutrition in Ruminants; Springer: Cham, Switzerland, 1990; ISBN 9781851664399.

49. Satter, L.D.; Slyter, L.L. Effect of ammonia concentration on rumen microbial protein production in vitro. Br. J. Nutr. 1974, 32, 199-208. [CrossRef]

50. Kholif, A.E.; Morsy, T.A.; Abdo, M.M. Crushed flaxseed versus flaxseed oil in the diets of Nubian goats: Effect on feed intake, digestion, ruminal fermentation, blood chemistry, milk production, milk composition and milk fatty acid profile. Anim. Feed Sci. Technol. 2018, 244, 66-75. [CrossRef]

51. Kholif, A.E.; Gouda, G.A.; Olafadehan, O.A.; Abdo, M.M. Effects of replacement of Moringa oleifera for berseem clover in the diets of Nubian goats on feed utilisation, and milk yield, composition and fatty acid profile. Animal 2018, 12, 964-972. [CrossRef] [PubMed]

52. Flatt, W.P.; Warner, R.G.; Loosli, J.K. Absorption of volatile fatty acids from the reticulo-rumen of young dairy calves. J. Dairy Sci. 1956, 39, 928.

53. Morsy, T.A.; Kholif, S.M.; Kholif, A.E.; Matloup, O.H.; Salem, A.Z.M.; Elella, A.A. Influence of sunflower whole seeds or oil on ruminal fermentation, milk production, composition, and fatty acid profile in lactating goats. Asian-Australas. J. Anim. Sci. 2015, 28, 1116-1122. [CrossRef] 
54. Martinez-Fernandez, G.; Denman, S.E.; Yang, C.; Cheung, J.; Mitsumori, M.; McSweeney, C.S. Methane inhibition alters the microbial community, hydrogen flow, and fermentation response in the rumen of cattle. Front. Microbiol. 2016, 7, 1122. [CrossRef]

55. Wild, K.J.; Steingaß, H.; Rodehutscord, M. Variability of in vitro ruminal fermentation and nutritional value of cell-disrupted and nondisrupted microalgae for ruminants. GCB Bioenergy 2019, 11, 345-359. [CrossRef]

56. Boeckaert, C.; Mestdagh, J.; Vlaeminck, B.; Clayton, D.; Fievez, V. Micro-algae as potent rumen methane inhibitors and modifiers of rumen lipolysis and biohydrogenation of linoleic and linolenic acid. Int. Congr. Ser. 2006, 1293, 184-188. [CrossRef]

57. Fievez, V.; Boeckaert, C.; Vlaeminck, B.; Mestdagh, J.; Demeyer, D. In vitro examination of DHA-edible micro-algae. 2. Effect on rumen methane production and apparent degradability of hay. Anim. Feed Sci. Technol. 2007, 136, 80-95. [CrossRef]

58. Newbold, C.J.; De la Fuente, G.; Belanche, A.; Ramos-Morales, E.; McEwan, N.R. The role of ciliate protozoa in the rumen. Front. Microbiol. 2015, 6, 1313. [CrossRef]

59. Dehority, B.A. Rumen Microbiology; Nottingham University Press: Nottingham, UK, 2003; ISBN 1897676999.

Publisher's Note: MDPI stays neutral with regard to jurisdictional claims in published maps and institutional affiliations.

(C) 2020 by the authors. Licensee MDPI, Basel, Switzerland. This article is an open access article distributed under the terms and conditions of the Creative Commons Attribution (CC BY) license (http://creativecommons.org/licenses/by/4.0/). 